\title{
ON SOME DIFFERENCE PROBLEMS
}

\section{TADEUSZ JANKOWSKI}

In this paper we discuss in some detail the difference equations arising in the discretization of some second-order differential equations. We also show how such difference problems can be solved exactly.

\section{INTRODUCTION}

Let $R^{p}$ denote the real $p$-dimensional space with the zero vector denoted by $\theta$. For real numbers $a, b, a<b$ and for $i=0,1$, let $C^{i}\left([a, b], R^{p}\right)$ denote the space of functions on $[a, b]$ into $R^{p}$ with continuous derivatives up to order $i$ and $C\left([a, b], R^{p}\right):=$ $C^{0}\left([a, b], R^{p}\right)$. Let $t_{n}=a+n h, n=0,1, \ldots, N$, where $N h=b-a$. Here $h$ is a constant stepsize.

In recent years there has been considerable interest in the theory and constructive methods for finding solutions of difference equations satisfying some boundary conditions. Difference problems appear in the study of discretisation methods for systems of differential equations (see $[1,2,3]$ ). As an example, let us consider a two-point boundary value problem of the form

$$
y^{\prime \prime}(t)=f(t, y(t)), \quad t \in J=[a, b], \quad y(a)=y_{a}, y(b)=y_{b},
$$

with $f \in C^{1}\left(J \times R^{p}, R^{p}\right)$. To find a numerical solution $y^{h}$ of (1) by the method of finite differences, we need to solve the difference problem

(2) $\left\{\begin{array}{l}y^{h}\left(t_{n}+h\right)-2 y^{h}\left(t_{n}\right)+y^{h}\left(t_{n}-h\right)=h^{2} f_{h}\left(t_{n}, y^{h}\left(t_{n}\right)\right), n=1,2, \ldots, N-1, \\ y^{h}(a)=y_{a}, \quad y^{h}(b)=y_{b},\end{array}\right.$

produced by applying a discretisation method. In this case, only the values of $y^{\boldsymbol{h}}$ at the mesh points $t_{n}$ are needed. It is well known that the solution of (2) is given by

$$
y^{h}\left(t_{n}\right)=\frac{n}{N} y_{b}+\left(1-\frac{n}{N}\right) y_{a}-h^{2} \sum_{i=1}^{N-1} d_{n i}^{\star} f_{h}\left(t_{i}, y^{h}\left(t_{i}\right)\right), n=0,1, \ldots, N
$$

Received 12th August, 1999

Copyright Clearance Centre, Inc. Serial-fee code: 0004-9727/00 \$A2.00+0.00. 
where

$$
d_{n i}^{\star}:= \begin{cases}i-\frac{i n}{N} & \text { if } i \leqslant n-1 \\ n-\frac{i n}{N} & \text { if } n \leqslant i\end{cases}
$$

Suppose we wish to use the method of finite differences to compute a numerical solution of the two-point boundary value problem for the system of deviated differential equations

$$
y^{\prime \prime}(t)=f(t, y(\alpha(t))), \quad t \in J, y(a)=y_{a}, y(b)=y_{b},
$$

with given $\alpha \in C(J, J)$. To do this it is necessary to have the values of $y^{h}\left(\alpha\left(t_{n}\right)\right)$ too.

In this case, we have to construct an algorithm for $y^{h}$ which makes it possible to compute the values of the approximate solutions at any point of the interval $J$. We set $\alpha(t)=a+h c(t)+h e(t)$ for fixed $t$, where

$$
c(t)=\left[\frac{\alpha(t)-a}{h}\right], \quad e(t)=\frac{\alpha(t)-a}{h}-c(t)
$$

and $[\cdot]$ denotes the integer part of the argument. In the above notation, we obtain

$$
y^{h}\left(\alpha\left(t_{n}\right)\right)=y^{h}\left(t_{c\left(t_{n}\right)}+h e\left(t_{n}\right)\right) \text { for fixed } n \text {. }
$$

We observe that $e: J \rightarrow[0,1)$.

A numerical solution of (4) based on the above may now be obtained by a difference method of the form

(5)

$$
\left\{\begin{array}{l}
y^{h}\left(t_{n}+r h\right)-y^{h}\left(t_{n}\right)-y^{h}\left(t_{n-1}+r h\right)+y^{h}\left(t_{n-1}\right)=g_{n}^{h}(r), n=1,2, \ldots, N-1, \\
y^{h}(a)=y_{a}, \quad y^{h}(b)=y_{b},
\end{array}\right.
$$

for $r \in[0,1]$ with $g_{n}^{h}(r):=h^{2} F\left(t_{n}, y^{h}, h, r\right)$ and $g_{n}^{h}(0)=\theta$. Here $F$ is an approximation to $f$. Note that algorithm (5) can be obtained by replacing the differential equation in (4) by the system of first-order differential equations

$$
\left\{\begin{array}{l}
y^{\prime}(t)=z(t) \\
z^{\prime}(t)=f(t, y(\alpha(t))), t \in J
\end{array}\right.
$$

and then using the approximations

$$
\left\{\begin{array}{l}
h z^{h}(t)=y^{h}(t)-y^{h}(t-h) \\
r h\left(z^{\prime}\right)^{h}(t)=z^{h}(t+r h)-z^{h}(t) .
\end{array}\right.
$$

The purpose of this paper is to provide a constructive way to present the solution of problem (5) in a form similar to (3). Some general properties will also be given. The problem of convergence of method (5) to the solution $y$ of (4) will be treated in a subsequent work. 


\section{Main Results}

In this section, we investigate the difference problem (5) and give a description of its solution. For given $g_{n}^{h}:[0,1] \rightarrow R^{p}, g_{n}^{h}(0)=\theta, n=1,2, \ldots, N$, we define the sequence $\left\{G_{k}\right\}$ by

$$
\left\{\begin{array}{l}
G_{1}(r):=g_{1}^{h}(r) \\
G_{k+1}(r):=G_{k}(1)+G_{k}(r)-G_{k}(0)+g_{k+1}^{h}(r), k=1,2, \ldots, N-1,
\end{array}\right.
$$

for $r \in[0,1]$. From the definition, we see that

$$
G_{k+1}(0)=G_{k}(1), \quad k=1,2, \ldots, N-1 \text {. }
$$

Lemma 1. Let $g_{n}^{h}(0)=\theta, n=1,2, \ldots, N$. Then the solution $y^{h}$ of (5) can be expressed as

$$
y^{h}\left(t_{n}+r h\right)=\frac{n}{N}\left(y_{b}-y_{a}-G_{N-1}(1)\right)+y^{h}(a+r h)+G_{n}(r)
$$

for $r \in[0,1]$ and $n=1,2, \ldots, N-1$.

Proof: By induction on $n$ it is simple to prove that

$$
y^{h}\left(t_{n}+r h\right)=n y^{h}\left(t_{1}\right)-n y_{a}+y^{h}(a+r h)+G_{n}(r)
$$

for $r \in[0,1]$ and $n=1,2, \ldots, N-1$.

Our next objective is to show that (6) holds. Take $n=N-1$ and $r=1$. From (7) we obtain

$$
y^{h}\left(t_{N}\right)=y_{b}=N y^{h}\left(t_{1}\right)-(N-1) y_{a}+G_{N-1}(1)
$$

and hence

$$
y^{h}\left(t_{1}\right)=\frac{1}{N}\left[y_{b}+(N-1) y_{a}-G_{N-1}(1)\right] .
$$

This and (7) yield (6).

REMARK 1. Relation (6) implies that

$$
y^{h}\left(t_{n}+h\right)=y^{h}\left(t_{n+1}+0 h\right)=\lim _{r \rightarrow 0^{+}} y^{h}\left(t_{n+1}+r h\right), \quad n=1,2, \ldots, N-1,
$$

provided that

$$
\lim _{r \rightarrow 0^{+}} G_{n}(r)=G_{n}(0) \quad \text { and } \quad \lim _{r \rightarrow 0^{+}} y^{h}(a+r h)=y_{a}
$$


REMARK 2. Let $p=1, g_{n}(r)=\sum_{i=1}^{n} a_{n, i} r^{i}, r \in[0,1], a_{n, i} \in R, n, i=1,2, \ldots, N$. From the definition of $\left\{G_{k}\right\}$,

$$
G_{k}(r)=\sum_{i=1}^{k} \sum_{j=i}^{k} a_{j, i}\left(k-j+r^{i}\right), \quad k=1,2, \ldots, N .
$$

After some calculation, the solution (6) of problem (5) takes the form

$$
y^{h}\left(t_{n}+r h\right)=\frac{n}{N}\left(y_{b}-y_{a}\right)+y^{h}(a+r h)-\sum_{i=1}^{N-1} \sum_{j=i}^{N-1} \bar{d}_{n, i, j}(r) a_{j, i}
$$

for $r \in[0,1], n=1,2, \ldots, N-1$, where

$$
\bar{d}_{n, i, j}(r)=\left\{\begin{array}{ll}
j-r^{i}-\frac{n j}{N} & \text { if } i \leq j \leq n, \\
n-\frac{n j}{N} & \text { if } n+1 \leq j \leq N-1
\end{array}\right\} 1 \leq i \leq n
$$

Indeed, if $a_{n, 1}=A_{n}$ and $a_{n, i}=0$ for $i=2,3, \ldots, n$ and $n=1,2, \ldots, N$, then we have immediately

$$
G_{k}(r)=\sum_{i=1}^{k}(k-i+r) A_{i}, \quad k=1,2, \ldots, N
$$

and in this case (8) assumes the form

$$
y^{h}\left(t_{n}+r h\right)=\frac{n}{N}\left(y_{b}-y_{a}\right)+y^{h}(a+r h)-\sum_{i=1}^{N-1} \tilde{d}_{n, i}(r) A_{i}
$$

with

$$
\tilde{d}_{n, i}(r)= \begin{cases}i-r-\frac{i n}{N} & \text { if } i \leqslant n \\ n-\frac{i n}{N} & \text { if } i>n .\end{cases}
$$

The next two lemmas give relations between $G_{n}$ and $g_{n}$. These should be useful for presenting the solution of problem (5) in a form similar to (3).

LEMMA 2. If $g_{n}^{h}(0)=\theta$, then for $n=1,2 \ldots, N$ and $r \in[0,1]$, we have

$$
G_{n}(r)=\sum_{i=1}^{n-1} i g_{n-i}(1)+\sum_{i=1}^{n} g_{i}(r)
$$

with $\sum_{i=1}^{0} \cdots=\theta$.

Proof: This formula can be proved by induction. 
REMARK 3. We note that

$$
G_{n}(1)=\sum_{i=1}^{n} i g_{n+1-i}(1), \quad n=1,2, \ldots, N
$$

results immediately from (9).

LEMMA 3. If $g_{n}^{h}(0)=\theta$, then

$$
-\frac{n}{N} G_{N-1}(1)+G_{n}(r)=\sum_{i=1}^{n} g_{i}(r)-\sum_{i=1}^{N-1} d_{n i}^{\star} g_{i}(1)
$$

holds for $n=1,2, \ldots, N$ and $r \in[0,1]$, where $d_{n i}^{*}$ are as in the introduction.

Proof: By Lemma 2 and Remark 3, we have

$$
\begin{aligned}
P: & =-\frac{n+1}{N} G_{N-1}(1)+G_{n+1}(r) \\
& =-\frac{n+1}{N} \sum_{i=1}^{N-n-1} i g_{N-i}(1)-\frac{n+1}{N} \sum_{i=N-n}^{N-1} i g_{N-i}(1)+\sum_{i=1}^{n} i g_{n+1-i}(1)+\sum_{i=1}^{n+1} g_{i}(r) .
\end{aligned}
$$

Furthermore, by a change of index in the first three sums, we get

$P=-\frac{n+1}{N} \sum_{i=1}^{n}(N-i) g_{i}(1)-\frac{n+1}{N} \sum_{i=n+1}^{N-1}(N-i) g_{i}(1)+\sum_{i=1}^{n}(n+1-i) g_{i}(1)+\sum_{i=1}^{n+1} g_{i}(r)$

whence we have (10). The proof is complete.

By Lemmas 1 and 3, expression (6) now takes the final form.

LEMMA 4. If $g_{n}^{h}(0)=\theta$, then

$$
y^{h}\left(t_{n}+r h\right)=\frac{n}{N}\left(y_{b}-y_{a}\right)+y^{h}(a+r h)+\sum_{i=1}^{n} g_{i}(r)-\sum_{i=1}^{N-1} d_{n i}^{\star} g_{i}(1)
$$

is the solution of (5) for $r \in[0,1]$ and $n=1,2, \ldots, N-1$.

\section{Some Comments}

Note that (11) is well-defined if the definition of $y^{h}$ is extended to $(a, a+h)$ so that (11) defines $y^{h}$ on $[a, b]$. For example, one might use the linear approximation

$$
y^{h}(a+r h)=(1-r) y_{a}+r y^{h}\left(t_{1}\right), \quad r \in[0,1],
$$


or the quadratic approximation

$$
y^{h}(a+r h)=\frac{1}{2}\left(r^{2}-3 r+2\right) y_{a}-\left(r^{2}-2 r\right) y^{h}\left(t_{1}\right)+\frac{1}{2}\left(r^{2}-r\right) y^{h}\left(t_{2}\right), \quad r \in[0,1],
$$

to define $y^{h}$ on the initial interval $(a, a+h)$. For example, if $y^{h}$ is defined by the above linear approximation on the interval $\left(t_{0}, t_{1}\right)$, then by (11) we see that $y^{h}$ is not a linear approximation between the values of $y^{h}\left(t_{1}\right)$ and $y^{h}\left(t_{2}\right)$ on $\left(t_{1}, t_{2}\right)$.

Note that $y^{h}$ is also well-defined by (11) if the definition of $y^{h}$ is extended to the interval $(b-h, b)$ instead of $(a, a+h)$. In this case, it is necessary to reformulate formula (11) and replace $y^{h}(a+r h)$ by $y^{h}\left(t_{N-1}+r h\right)$.

REMARK 4. The difference problem (5) may also be solved for a fixed value of $r \in(0,1]$ only. If $r=1$, then it is well-known that the solution of (5) has the form (3). In this case (11) reduces to (3). If $r$ is fixed and $r \in(0,1)$, then we need only the value $y^{h}(a+r h)$ to solve (5) (see (11)).

\section{REFERENCES}

[1] R.P. Agarwal, Difference equations and inequalities. Theory, methods, and applications (Marcel Dekker, New York, 1992).

[2] U.M. Ascher, R.M.M. Mattheij and R.D. Russell, Numerical solution of boundary value problems for ordinary differential equations (Prentice Hall, New Jersey, 1988).

[3] V. Lakshmikantham and D. Trigiante, Theory of difference equations (Academic Press, Toronto, 1988).

Department of Differential Equations

Technical University of Gdansk

11/12 Narutowicz Str.

80-952 Gdansk

Poland

e-mail: tjank@mifgate.pg.gda.pl 